synovitis, lymphadenopathy, nodules, a sensory neuropathy, scleritis, and extensive nailfold vasculitis.

Ophthalmological examination including rose bengal staining confirmed sicca syndrome, and bilateral active scleritis with scleral thinning was also noted. Also the right retina showed a background pigmentary disturbance and several large fresh soft white exudates randomly distributed over the posterior pole. No retinal changes were seen in the left eye. Her right visual acuity was $6 / 18$. At an ophthalmic examination six months earlier both retinae were normal and the right visual acuity $6 / 9$.

Investigations showed haemoglobin concentration $14.5 \mathrm{~g} / \mathrm{dl}$, viscosity $2.23 \mathrm{mPa} \mathrm{s}(2.23 \mathrm{cP})$, rheumatoid factor titre $1 / 2560$, and antinuclear factor titre $1 / 40 . \mathrm{C}_{3}$ and $\mathrm{C}_{4}$ values were normal, though there was evidence of high concentrations of circulating immune complexes: anticomplementary activity $1 / 32$ (normal 1/2). Clq binding was $65 \%$ (normal $20 \%$ ), platelet aggregation $1 / 512$ (normal $1 / 16$ ), and cryoglobulins positive. Chest radiography showed extensive old tuberculosis but results of sputum culture and microscopy were negative.

The patient was treated with bed rest, non-steroidal anti-inflammatory drugs, local hypromellose and cortisone eye drops. After two months there was a noticeable improvement, and by six months the retinal changes had disappeared and visual acuity had improved. Her systemic rheumatoid disease also became less active, she gained weight, and tests for circulating immune complexes became negative.

\section{Discussion}

Retinal vasculitis may complicate many systemic diseases, ${ }^{1}$ including tuberculosis, sarcoidosis, Behçet's syndrome, and systemic lupus erythematosus. In a recent review ${ }^{2}$ of 17 patients with retinal vasculitis, immune complexes were found in the sera of 13 and rheumatoid factor was present in four.

Vasculitis complicating rheumatoid arthritis ${ }^{34}$ usually occur及 in nodular disease, with high titres of classical $\operatorname{IgM}$ rheumatoi\$ factor and raised concentrations of circulating immune com $\longleftarrow$ plexes. Infarcts in nailfold capillaries are often seen and weigh( loss and general malaise are common. Our patient had all these $\overline{\bar{e}}$ features, including circulating immune complexes detected $b \not{D}$ four different assays. The timing of the retinal disease and the observed rapid clinical improvement that accompanied the fae in concentrations of circulating immune complexes suggest that: the retinal changes were a direct consequence of her activi rheumatoid disease.

We suggest that the retina should be examined for evidence of vasculitis in rheumatoid disease, and that vasculitis should added to the list of ocular complications of rheumatoid arthritis.

\section{References}

${ }^{1}$ Saunders MD. Retinal vasculitis: a review. 7 R Soc Med 1979;72:908-1

2 Andrews BS, McIntosh J, Petts V, Penny R. Circulating immune comb plexes in retinal vasculitis. Clin Exp Immunol 1977;29:23-9.

${ }^{3}$ Fauci AS, Haynes BF, Katz P. The spectrum of vasculitis. Ann Inter Med 1978;89:660-76.

4 Scott DGI, Tribe CR, Bacon PA. Systemic rheumatoid vasculitis: clinicâ and laboratory studies in 50 patients. Medicine (Baltimore) (in press?

${ }^{5}$ Murray McGavin DD. Episcleritis and scleritis. In: Buchanan WW Dick WC, eds. Recent advances in rheumatology. Vol 1. Part II. London: Churchill Livingstone, 1976:166-92.

(Accepted 14 April 1981)

\title{
Haloperidol secreted in breast milk
}

\section{J WHALLEY, P G BLAIN, J K PRIME}

\begin{abstract}
A nursing mother was given haloperidol $5 \mathrm{mg}$ twice daily for puerperal psychosis and continued to breast feed under hospital supervision. Despite considerable amounts of haloperidol being secreted in the breast milk (up to $23.5 \mu \mathrm{g} / 1$ ), the infant was apparently not sedated, fed well, and continued to thrive.

The findings suggest that maternal ingestion of haloperidol for short periods has no deleterious effect on the infant's development.
\end{abstract}

\section{Introduction}

Mania occurring in the puerperium, though rare, can present important problems of management, particularly when the mother continues to breast feed. We report a case of puerperal psychosis treated with haloperidol in which substantial amounts of the drug were secreted in breast milk and ingested by the infant. There was no evidence, however, of any long-term effect on the infant's development.

\footnotetext{
MRC Brain Metabolism Unit, Thcmas Clouston Clinic, Edinburgh EH10 5LG

L J WHALLEY, MD, MRCPSYCH, honorary consultant psychiatrist

Wolfson Unit of Clinical Pharmacology, Newcastle upon Tyne P G BLAIN, MRCP, Wellcome research fellow

GP Practice, 38 Palmerston Place, Edinburgh EH12 5BJ

J K PRIME, RGN, SCM, health visitor
}

\section{Case report}

A mother delivered a live girl weighing $2660 \mathrm{~g}$ at 39 week's gestation after induction of labour with an intravenous infusion of oxytocin Apgar scores were 9 at one and five minutes. Pregnancy had bee? uneventful and there was no history of psychiatric illness. The immediate postnatal period progressed normally; breast feeding was established and mother and child were well. On the 16th day the mother was found by the health visitor to be tense, anxious, ang talking continuously. The general practitioner diagnosed puerpera्यु psychosis and prescribed chlorpromazine $25 \mathrm{mg}$ four times daily There was no improvement, and on the 20th day mother and bab疋 were admitted to hospital. The baby was progressing well and breas feeding was continued under supervision. The mother was deludec o euphoric, and overactive. Puerperal hypomania was diagnosed an@ treatment begun with oral haloperidol $5 \mathrm{mg}$ twice daily.

Samples of the mother's plasma and breast milk and the baby's urin were collected on the first, sixth, seventh, and 21 st days of treatmenn Only one of the urine samples was suitable for assay. The concentrart tion of haloperidol in these samples was measured by radioimmuno assay (sensitivity $0.5 \mu \mathrm{g} / \mathrm{l}$ ). The table gives the results.

Four weeks after the birth mother was improving, though still vere tense and anxious. The baby did not appear to be sedated and wa\$ feeding well. Haloperidol was stopped in the sixth week after birth an

\begin{tabular}{|c|c|c|c|c|}
\hline Day & Dose & Mother's plasma & Mother's milk & Baby's urine \\
\hline $\begin{array}{r}1 \\
6 \\
7 \\
21\end{array}$ & $\begin{array}{l}\text { None } \\
5 \mathrm{mg} \text { twice daily } \\
5 \mathrm{mg} \text { twice daily } \\
\text { None }\end{array}$ & $\begin{array}{r}0 \\
40 \\
26 \\
4\end{array}$ & $\begin{array}{l}0 \\
23 \cdot 5 \\
18 \cdot 0 \\
3 \cdot 25\end{array}$ & $\frac{0}{1.5}$ \\
\hline
\end{tabular}


mother and baby discharged home. At five months breast feeding was discontinued. The mother remained well and the baby had achieved all her expected developmental milestones at six months and one year. At no time did we observe any behavioural or motor abnormalities.

\section{Discussion}

The secretion of haloperidol in breast milk has been reported ${ }^{1}$ but the amount of drug ingested by the breast-fed infant has not been measured. Our results confirm that appreciable amounts of haloperidol may be secreted in breast milk and thus ingested by the infant.

Adverse behavioural effects may occur in the young of nursing animals given haloperidol. ${ }^{2}$ There have been no reports of adverse effects in infants breast fed by mothers taking haloperidol, though such infants apparently receive a substantial amount of the drug. In our case the baby's development was closely monitored and no behavioural abnormalities were noted. Normal developmental milestones were reached by the age of one year.

Our results suggest that, though haloperidol when administered in therapeutic doses is secreted in breast milk, ingestion for less than three weeks does not have a deleterious effect on the infant's development.

We thank Dr A I M Glen and Dr H H Gebbie for permission to study a patient under their care.

\section{References}

1 Stewart RB, Karas B, Springer PK. Haloperidol excretion in human milk. Am f Psychiatry 1980;137:849-50.

${ }^{2}$ Lundborg P. Abnormal ontogeny in young rabbits after chronic administration of haloperidol to the nursing mothers. Brain Res 1972 $44: 684-7$.

\title{
Malaria diagnosis by enzyme-linked immunosorbent assays
}

\author{
DENNIS E BIDWELL, ALISTER VOLLER
}

\begin{abstract}
Enzyme-linked immunosorbent assays (ELISA) for detecting malarial parasites in blood were tested in uninfected monkeys and in monkeys infected with Plasmodium falciparum. A double antibody sandwich ELISA detected one malarial cell per $10^{3}$ uninfected cells, and an inhibition ELISA detected one infected cell per $10^{4}$ uninfected erythrocytes.

These methods are not yet as sensitive as conventional blood-film examinations, in which a well-trained microscopist might be expected to detect one malarial cell per $10^{6}$ erythrocytes. Nevertheless, ELISA offers an objective means of detecting malaria and is particularly advantageous since up to 100 samples may be processed simultaneously.
\end{abstract}

\section{Introduction}

The conventional way of diagnosing malaria is by visual microscopical detection of malaria parasites in stained blood films. This is an efficient procedure if well-trained staff are available, when very low parasitaemias may be detected. Malaria is only rarely encountered in most pathological laboratories, however, and parasites are often overlooked. Even in areas where malaria is endemic blood-smear examination may be unreliable unless a system of random checking is implemented.

Immunoassay is an attractive and effective way of measuring antigens and antibodies. The methods are specific and sensitive, and often the same method may be used for a wide variety of applications. Routine screening of blood for hepatitis B surface

\footnotetext{
Nuffield Laboratories of Comparative Medicine, Institute of Zoology, Zoological Society of London, London NW1 4RY

DENNIS E BIDWELL, PHD, research assistant

Department of Clinical Tropical Medicine, London School of Hygiene and Tropical Medicine, London WC1 7HT

ALISTER VOLLER, DSC, MRCPATH, reader in immunology of parasitic diseases
}

antigen shows that immunoassays may be done efficiently on a large scale. For malaria, most immunoassays have been set up for the measurement of malarial antibody values, ${ }^{1}$ which may yield valuable epidemiological data on malaria transmission. Several years ago extensive studies were carried out on the type and prevalence of Plasmodium falciparum antigens in West Africa, ${ }^{2}$ but the purpose of the studies was analytical and the methods were not designed to detect slight parasitaemia. More recently isotopic methods have been used successfully to assay malarial antigens in the blood of experimentally infected animals ${ }^{3}$ and of naturally infected people. ${ }^{4}$ Radioimmunoassay techniques are not, however, suitable for use in most parasitological laboratories. This paper considers the use of enzyme-linked immunosorbent assays to detect $P$ falciparum parasites in blood.

\section{Materials and methods}

Antiserum -A high-titre antiserum to $P$ falciparum was obtained from an aotus monkey that had been infected and repeatedly challenged with $P$ falciparum (Palo Alto). The immunoglobulin fraction of the serum was prepared for coating microplates and part of it labelled with horse-radish peroxidase as described previously. ${ }^{5}$

Antigen-Aotus monkeys were infected with $P$ falciparum, and when parasitaemias were $20 \%$ or above blood was taken into heparin. The erythrocytes were separated by centrifugation and washed free of plasma. Soluble malarial antigen was obtained by sonic disruption of the infected erythrocytes. ${ }^{6}$

Test samples-Peripheral blood samples were collected into heparinised capillary tubes from uninfected saimiri monkeys and saimiri monkeys infected with $P$ falciparum. Blood films were made at the same time and stained with Giemsa.

ELISA

Two versions of microplate ELISA were used-namely, the double antibody sandwich and the inhibition methods. Full technical details of these have been reported. ${ }^{5}$

Double antibody sandwich ELISA-Polyvinyl microplates were coated with immunoglobulin $(10 \mu \mathrm{g} / \mathrm{ml})$ from the antimalarial serum. The plates were then coated with $5 \%$ bovine serum albumin. After washing the plates were dried and then sealed in aluminium foil pouches in which they were stored at $4^{\circ} \mathrm{C}$. 